\title{
2-Step Regularization on Style Optimization for Real Face
} Morphing

This paper was downloaded from TechRxiv (https://www.techrxiv.org).

LICENSE

CC BY-NC-SA 4.0

SUBMISSION DATE / POSTED DATE

02-02-2022 / 10-02-2022

\section{CITATION}

Yu, Cheng; Wang, Wenmin; Lei, HongLei; Bugiolacchi, Roberto (2022): 2-Step Regularization on Style Optimization for Real Face Morphing. TechRxiv. Preprint. https://doi.org/10.36227/techrxiv.19105493.v1

DOI

10.36227/techrxiv.19105493.v1 


\title{
2-Step Regularization on Style Optimization for Real Face Morphing
}

\author{
Cheng Yu ${ }^{\mathrm{a}, \mathrm{b}}$, Wenmin Wang ${ }^{\mathrm{b}, \mathrm{c}, *}$, Honglei Li ${ }^{\mathrm{b}}$, Roberto Bugiolacchi ${ }^{\mathrm{c}, \mathrm{d}}$ \\ ${ }^{a}$ Faculty of Information Technology, Macau University of Science and Technology, Macau 999078, China \\ ${ }^{b}$ International Institute of Next Generation Internet, Macau University of Science and Technology, Macau 999078, China \\ ${ }^{c}$ State Key Laboratory of Lunar and Planetary Sciences, Macau University of Science and Technology, Macau 999078, China \\ ${ }^{d}$ University College London, London WC1E 6BT, United Kingdom
}

\begin{abstract}
As a class of generative models, generative adversarial networks (GANs) are now capable of achieving excellent results, especially relating to facial images, which could reach $1024 \times 1024$ resolution in high-quality face synthesis. Recently, some studies have tried to map real face images into the latent space of GANs, for pre-trained GANs to represent real faces (E.g., optimizing style latent space on StyleGANs). However, the application of latent space to real face morphing is still in its infancy. Currently, training costs are high and/or require huge samples with labels. By adding regularization to the latent optimization, we propose a novel method to morph real faces based on StyleGAN. We labelled 12,000 face images with 32 attributes and utilise logistic-regression models to discover independent attribute vectors in latent space. Appropriate regularization helps us to regularize both latent vectors (a face with its attribute) so that they can fall into an ideal area. Moreover, we use those attribute vectors under different layer representations to handle real face morphing. Compared to the existing baseline, our method yields a larger output with higher quality.
\end{abstract}

Keywords: Face Morphing, Logistic Regression (LR), Generative Adversarial Net (GAN), Latent Representation

\section{Introduction}

Generative adversarial networks (GANs) [1] have made a big breakthrough in high-quality (HQ) image synthesis, especially on human face datasets [2, 3]. Compared to most autoencoders, GAN trains the generator with a partner, the discriminator, which makes GAN reach a better performance. When a convolutional neural network (CNN) is firstly utilised in deep GANs (DCGAN) [4], adopting deeper networks with stable training skills become the main trend in state-of-the-art (SOTA) GANs, such as PGGAN [5], BigGAN [6], StyleGANs [3, 7, 8]. Although SOTA GANs could yield high quality (HQ) synthesized images, training deep GANs requires a substantial investment in both hardware and time: consequently, utilising pretrained deep GANs for image representation could save costs and represent a considerable improvement. How to use deep GANs to represent and control a real face? To answer this question, we focus on face image morphing via StyleGANs.

Most GANs use normal latent space for representation that only takes one layer as input. Furthermore, in StyleGANs the decoupling of style latent space produces better performance. StyleGANs are better at representing synthesized images by the low-dimensional style latent space. Based on StyleGANs, current works try to map real face images [9, 10, 11] into latent space; discovering attribute vectors in latent space is also valu-

\footnotetext{
${ }^{*}$ Corresponding author.

Email addresses: disanda@outlook.com (Cheng Yu), wmwang@must .edu.mo (Wenmin Wang), Honglei_li@qq.com (Honglei Li), roberto.bugiolacchi@ucl.ac.uk (Roberto Bugiolacchi)
}

able to research. In essence, two approaches are utilised to discover attribute vectors. The first is the supervised manner [12] that utilises support vector machine (SVM), and depends on large-scale face images, latent vectors, and labels. The second is the unsupervised manner [12, 13] that utilises principal component analysis (PCA) to find potential attribute vectors that could perform as semantic directions.

However, the above methods have several limitations. The supervised method [14] needs large-scale attribute labels, while the unsupervised method [12,13] requires observing most principal components to find usable directions. Both methods incur huge costs (both in time and hardware) to optimize attribute vectors. Meanwhile, in face images, they only handle limited attributes and are not smooth enough for real face morphing. In this paper, we strive for real face morphing with richer attributes via limited labels.

In comparison to the work carried out on image reconstructions [9, 11], we analyse face images to latent vectors with regularizations along with different linear models. This is a different approach from [14], which utilises SVM for attribute classification: we revisit linear models with different regularizations [15, 16] and use logistic regression (LR) with $e l_{1}$-norm to save both time and training labels. Finally, we offer a new method based on 2-step regularization that can make usable coefficient weights fast for face attribute change.

[17] and [18] show that different GAN layers and units could represent different semantic objects. Inspired by [17], we analyse different GAN layers to represent different attributes. We divide attributes into two categories (texture and colour), and 
we also divide the StyleGAN generator into 5 different layer levels according to the depth (deep to shallow) of the whole model layers. We operate the latent attribute vectors according to the corresponding layers levels to discover latent style vectors to control face morphing. The specific layer levels could represent more and finer attributes for real face morphing.

In addition, we analyse two famous face attribute classifiers (from face libraries of Microsoft and Nvidia), throw out the invalid labels, while keeping the valid ones. Our method derives 33 attributes from face labels and can be applied to real face morphing to produce vivid animations. (See Fig. 1)

To summarize, we describe the contributions thus:

- We propose a novel approach to make attribute vectors via StyleGANs for real face morphing. To the best of our knowledge, it is the first method that utilises limited labels (within 8,000) to find usable attribute vectors. Compare with the baseline, it is a fast and simple way to achieve acceptable face morphing results.

- Norm values of latent vectors are the key to our discovery in latent representation. For face attribute morphing we offer a 2-step regularization to control norm values. One norm is face vectors for real faces, and the other is to attribute vectors for semantic directions. For face vectors, we divide them into four areas and give an ideal area for attribute representation. For attribute vectors, we offer a simple method for trimming a specific range to decouple attribute interconnections.

- We analyse the relationship between the face attributes and layer-wise representation, and we give a guideline for real face morphing. Meanwhile, we collect 33 usable attribute labels from two attribute classifiers. Both on quantity and quality indexes, these labels could yield excellent face attribute changes.

\section{Related Work}

\subsection{Latent Representation}

In the generative model, latent representation is the relationship between the low-dimensional vectors and the image semantic attributes during image generation. Crucially, HierarchyGAN [17] found that GANs can represent different semantic laws in different layers. E.g., a normal latent vector input model and then output an image. Each layer in the model could orderly represent images of 'layout', 'whole objects', 'object attributes' and 'colour scheme'. Similarly, GAN-Dissection [18] demonstrates through PGGAN that different units in the same layer can represent different object types and corresponding regions of the synthesized images. However, these studies only worked on LSUN dataset [19] for pre-trained GANs. We expand the rule of layer-level representation on the face dataset [2] and finally utilise the rule for morphing real faces.

\subsection{StyleGAN Embedding}

StyleGANs have achieved high performance on face images and also better representation via style latent vector, $w$. Compare with normal latent vector $z, w$ is a layer-wise vector that can represent each layer of GAN. Image2StyleGAN [9] attempts to map real images into $w$ via directly optimizing $w$, but directly optimizing $w$ is an inefficient approach marred with low performance when dealing with real faces. Deep GANEncoders [20, 21, 11] try to encode images into $w$ via autoencoder with additional optimizing $w$. Currently, the above works could map real faces to $w$, however, how to represent semantic directions via $w$ remains to be explored.

\subsection{Real Face Morphing}

Based on StyleGAN embedding, we can get the real face representation via $w$. Next, we also need to learn the usable directions on $w$ and move those directions for the real face morphing. There are two manners to deal with this.

One is the unsupervised manner, no labels are required. The methods, GANSpace [12] and EigenGAN [13], should train on huge samples to find the principal vectors on $w$. Those vectors could represent semantic attributes. However, most vectors are invalid or redundant, so the methods should check each vector carefully. GANSpace trains on $w$ and can perform on pre-trained models. EigenGAN trains the models with a set of orthogonal vectors.

The other one is the supervised manner. The method (InterfaceGAN [14]) needs samples with labels. Based on SVM, the method needs huge samples with labels. It can find a boundary on each label and regard the orthogonal boundary as the label direction. However, most boundaries are coupled on the semantic attribute, so InterfaceGAN uses another boundary to alleviate the coupling. But moving two boundaries on latent space is unstable. It is only valid on a part of cases.

\section{Approach}

\subsection{Problem Description}

\subsubsection{Basic Explication}

$z$ denotes the normal latent vector whose element size is 512 . For training GANs, $z$ usually represents the multivariate normal distribution, which is treated as the prior distribution. $x$ denotes synthesized face image and $y$ denotes real face image. Both sizes $(x$ and $y)$ are pixel-wise, and the element size is $(3,1024$, 1024).

We use pre-trained StyleGAN [3] for performing our method. $w$ denotes the latent style vector, and its size is $(18,512)$. Here, $w$ is layer-size latent vector. $w$ is divided into 18 layers throughout whole generators, and each layer have 512 dimensions. $M$ denotes the mapping networks and it maps $z$ to $w$, i.e., $w=M(z)$. $G$ denotes the generator and it can synthesize $1024 \times 1024$ face images, i.e., $x=G(w)$.

$d$ denotes the label for a specific attribute direction. We performed $d$ as a one-hot vector. $d$ indicates the positive correlation for the attribute, and $-d$ indicates the negative correlation. It is planned to optimize the coefficient weight of $d$ in $w$ through 


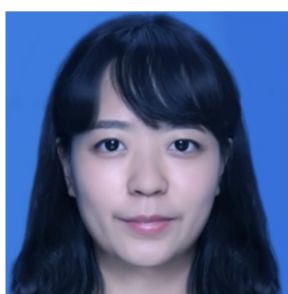

Real Image

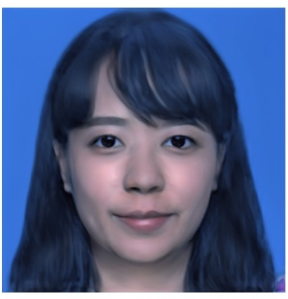

Black Skin

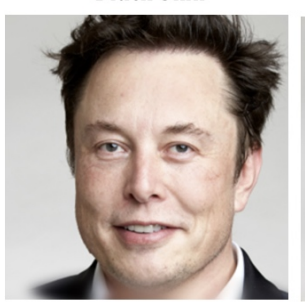

Real Image

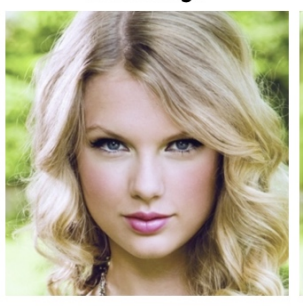

Real Image

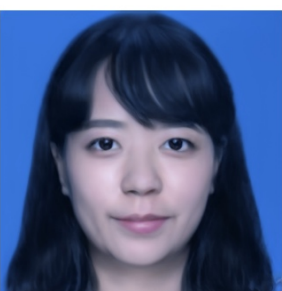

Reconstruction

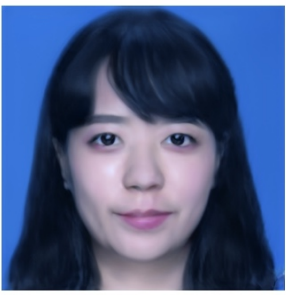

Eyelid

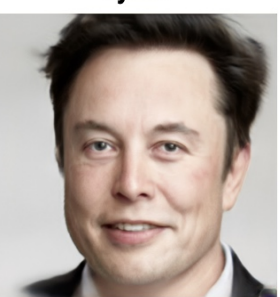

Reconstruction

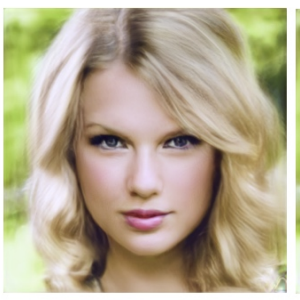

Reconstruction

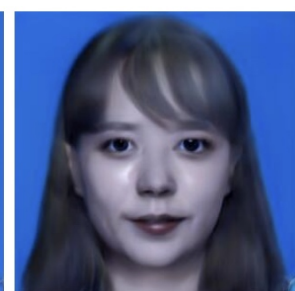

Blond Hair

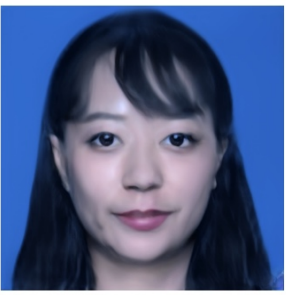

Bald

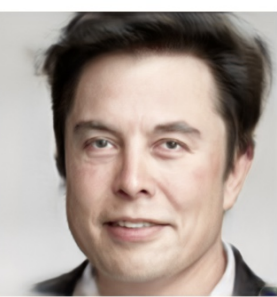

Hair

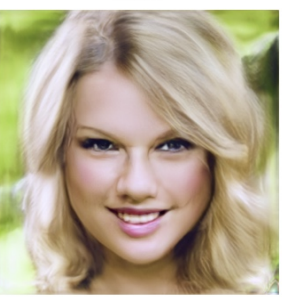

Smile

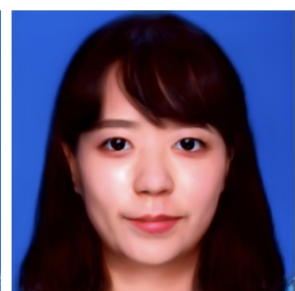

Red

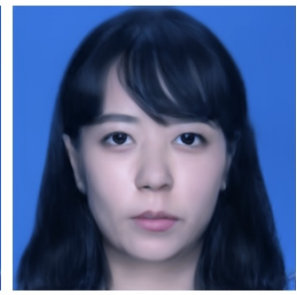

Arrogant

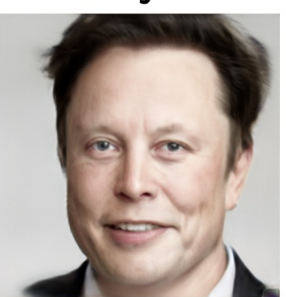

Eyebrow

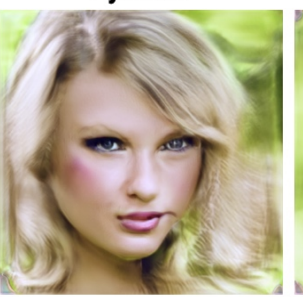

Pose Left

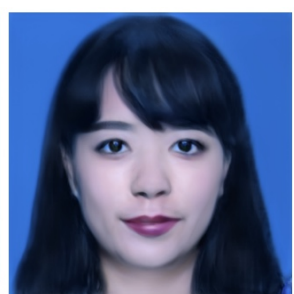

Makeup

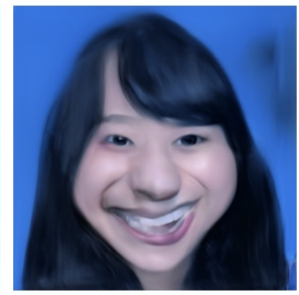

Comedy

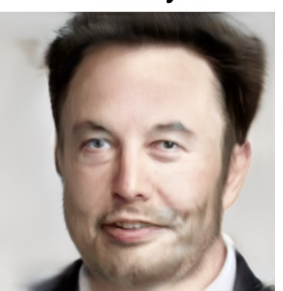

Beard

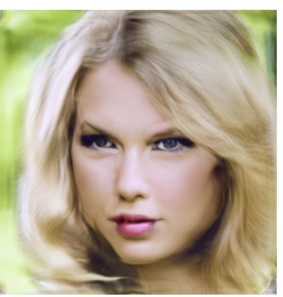

Pose Right

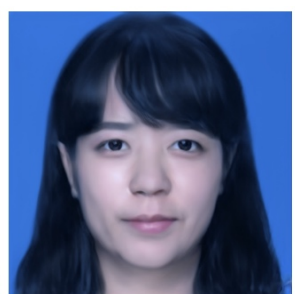

Gender

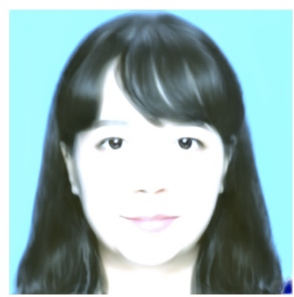

Over Exposure

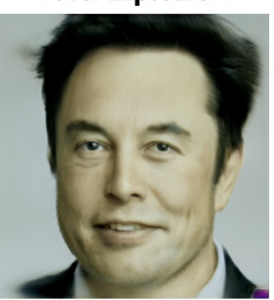

Dark

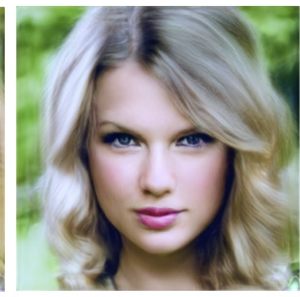

Black Hair

Figure 1: The above images show the morphing of real human faces. Based on StyleGAN [3], we utilise [11] that can embed real face images into style vectors. Meanwhile, we use limited labels $(8,000)$ for each attribute vector to morph real images. Specifically, where image 'Red' $(1$ st row) is from the attribute 'Red Hair', and image 'Hairs' (3rd row) is from the attribute 'Hairline'. Image 'Over Exposure' (2nd row) and Image 'Dark' (3rd row) are from the same attribute 'Exposure' (Opposite directions). Similarly, image 'Comedy' (2nd row) and image 'Arrogant' (2nd row) are also from the same attribute 'happy'.

linear classification models. Here, $w_{d}$ denotes the coefficient weight which is the same size as $w$. We consider that the orthogonal direction of $w_{d}$ is the attribute direction. Here, we call $w_{d}$ the attribute vector for short.

$w_{y}$ is same size with $w$ which could represent a real image $y$ in style latent space. We called $w_{y}$ as the face vector for short. Following on [11], we use a pre-trained encoder $(E)$ to map $y$ into $w_{y}$, then we get a synthesized image $x_{y}$ which is similar with $y$. That is, based on $w_{y}=E(y)$, and then we get $x_{y}=G\left(w_{y}\right)$ , and $x_{y} \simeq y . w_{y}$ needs $E$ and the further optimization as follows:

$$
\mathcal{L}_{w_{y}}=\arg \min _{w_{y}} \sum_{i=1}^{n}\left(L_{1}\left(G\left(w_{y_{i}}\right), y\right)+\mathcal{L}_{2}\left(w_{y_{i}}, E(y)\right)\right)
$$

Where the loss function $\mathcal{L}_{1}$ is used to measure the difference of the real image with its reconstruction $\left(y\right.$ and $x_{y}$ ), and the loss function $\mathcal{L}_{2}$ is used to measure the difference of the encoded $w$ (from $E(y)$ ) with optimizing $w_{y}$. Finally, $x_{d}$ denotes the morphed real image which is represented by the direction $d$ as follows:

$$
x_{d}=G\left(w_{y}+\alpha w_{d}\right)
$$

Here, $\alpha$ is the distance parameter of $w_{d}$.

\subsubsection{Key Observation}

Depending on the different samples and labels, as well as different classification methods, the $w_{d}$ found is likely to be different. Testing through a large number of samples, it emerged that the coefficient weights of the different attributes are orthogonal to each other. Furthermore, we found that the coefficient weights obtained by different methods for the same attribute are also orthogonal. Therefore, we consider that there exist multiple solutions for the $w_{d}$ for the same attribute $d$. However, these solutions via linear classify models are coupled or even unusable for face attribute morphing.

As shown in Fig. 2 (a), when looking for simple attribute 


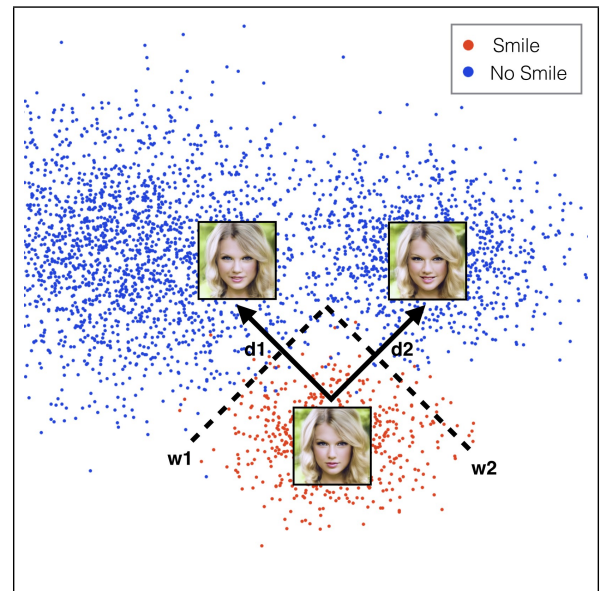

(a)

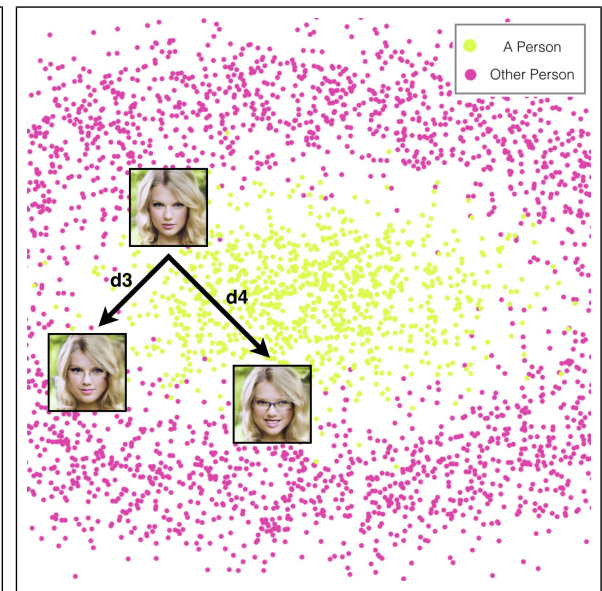

(b)

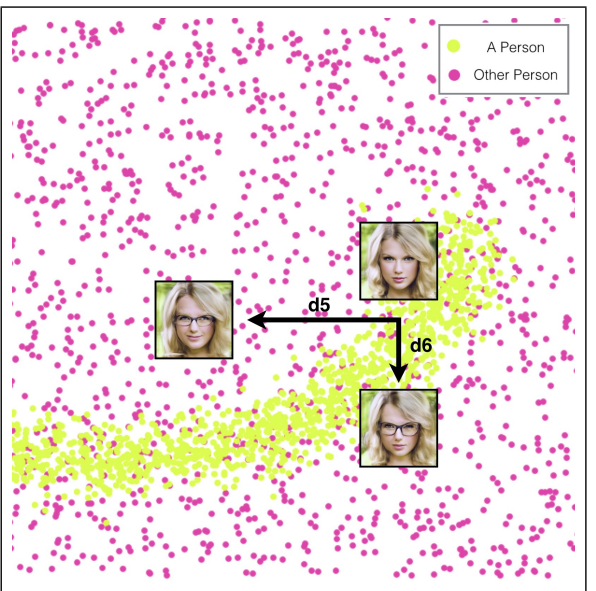

(c)

Figure 2: There are 3 occasions for the attribute change. Both smile directions $\left(d_{1}\right.$ and $\left.d_{2}\right)$ can make real face smile (a). However, different glasses directions $\left(d_{3}\right.$ $d_{6}$ ) yield attribute coupling on different cases. $d_{3}$ changes to another person. $d_{4}$ is coupling with smile. $d_{5}$ and $d_{6}$ are coupling with age (younger or older).

directions' $w_{d}$ they may be all valid, for instance, the smile directions $\left(d_{1}\right.$ and $\left.d_{2}\right)$. However, searching for usable $w_{d}$ is complicated on most attribute directions, such as the directions of eyeglasses, these are likely to make a difference of attribute coupling, e.g, $d_{4}$ is coupling with smile. $d_{5}$ is coupling with old. $d_{6}$ is coupling with young, and even this direction is invalid $\left(d_{3}\right)$.

When we move $w_{d}$ to a short distance, most of the attribute changes remain valid $(\alpha<100)$. As the distance increases, most attributes stay coupled on image morphing, thus our goal shifts to finding the decoupling way to move individual attributes to a longer distance.

According to the training strategy of StyleGAN, we observe that the $w$ obeys a Gaussian distribution (same with $w_{y}$ and $w_{d}$ ), which means that their value of mean $\mu$ is 0 . We consider that this represents an original point. All element values of the original point are 0 , i.e., $\ell_{2}-$ norm $\left(\|w\|_{\rho=2}=0\right)$. The other face and attribute changes are the specific distances based on the original point.

\subsection{The First Regularization for Face Vector}

We denote Norm as the value of $\ell_{2}-$ norm $\left(\left\|w_{y}\right\|_{\rho=2}\right)$. As shown in Fig. 3, the original point is $N o r m=0$ (red point). We use pose attribute as the test direction. We divide the value range of $w_{d}$ into four regions $\left(\right.$ Zone $_{1}-$ Zone $\left._{4}\right)$ according to different Norm. While Norm $\approx 0$, the real face identity is essentially similar to the original point.

If $w_{y}$ falls into Zone ${ }_{1}, w_{y}$ cannot even represent the real face $y($ Norm $=98)$ and adding $w_{d}$ will make it sensitive to real face morphing. With the increasing of Norm, and dropping into Zone $_{2}-$ Zone $_{3}$, the real face identity can be represented well, i.e, $x_{y} \simeq y$, but the sensitivity of attribute changing will also be decreasing. When Norm is too large (dropping into Zone ${ }_{4}$ ), although $w_{y}$ can fully represent the identity of $y, w_{d}$ becomes unable to change attributes for real face morphing. This phenomenon is similar to model collapse.

To find a compromise to the representations of $w_{d}$ and $w_{y}$, we attempt to get Norm of $w_{y}$ in Zone 3 . Let $w_{y}$ fully repre- sent real face image and $w_{d}$ could morphing attribute smoothly. Consequently, we upgrade Eq. 1, adding a regularization term:

$$
\mathcal{L}_{w_{y}}=\arg \min _{w_{y}} \sum_{i=1}^{n}\left(\mathcal{L}_{1}\left(G\left(w_{y_{i}}\right), y\right)+\mathcal{L}_{2}\left(w_{y_{i}}, E(y)\right)\right)+\beta\left\|w_{y}\right\|_{\rho=2}
$$

For ensuring $w_{y}$ drops into $Z_{o n e_{3}}$, we use parameter $\beta=0.01$ and $\ell_{2}-$ norm for regularization. Next section will discuss these issues in more detail.

\subsection{Layer-Wise Moving}

As shown in Fig. 4, we get $w$ from $z$ which passes through mapping network $M$. By truncating the first 8 layers towards original point $(N o r m=0)$, we get $w_{\text {trunc }}$, i.e., $w_{\text {trunc }}=0.7 w$ for the first 8 layers. Compared to $w_{d}, w_{\text {trunc }}$ is closer to original point, and better represent the face identity. Next, we get $w_{d}$ and a bias $b$ via linear models. That could be optimized as follows:

$$
\mathcal{L}_{w_{d}}=\arg \min _{w_{d}, b} \sum_{i=1}^{n} \mathcal{L}\left(w_{x_{i}}, d_{i}, w_{d}, b\right)+\gamma\left\|w_{d}\right\|_{\rho}
$$

HierarchyGAN [17] found that in the bedroom dataset of LSUN [19], layers of different depths in StyleGAN have different representatives. We tested 32 face attributes and found that styleGAN has similar patterns in the face dataset [2, 3].

As shown in Fig. 4, we follow the laws of attributes, divide layers into two categories (texture and colour) and divide them into five layer-levels (L1-L5) according to the network layer from deep to shallow, where texture represents the changes of the face and contains L1-L3. Here, L1 is mainly the attribute of high-level statements, representing the whole facial changes, such as smile, glasses, expression, etc. L2 represents local changes of the face, such as the beard, hair, eyes, lips. L3 is more related to whole face colours, such as hair colours. In shallower layers, L4-L5, represent whole image colours, with the difference that L4 mainly highlights the colours of the face, while L5 highlights the colours of the background. 


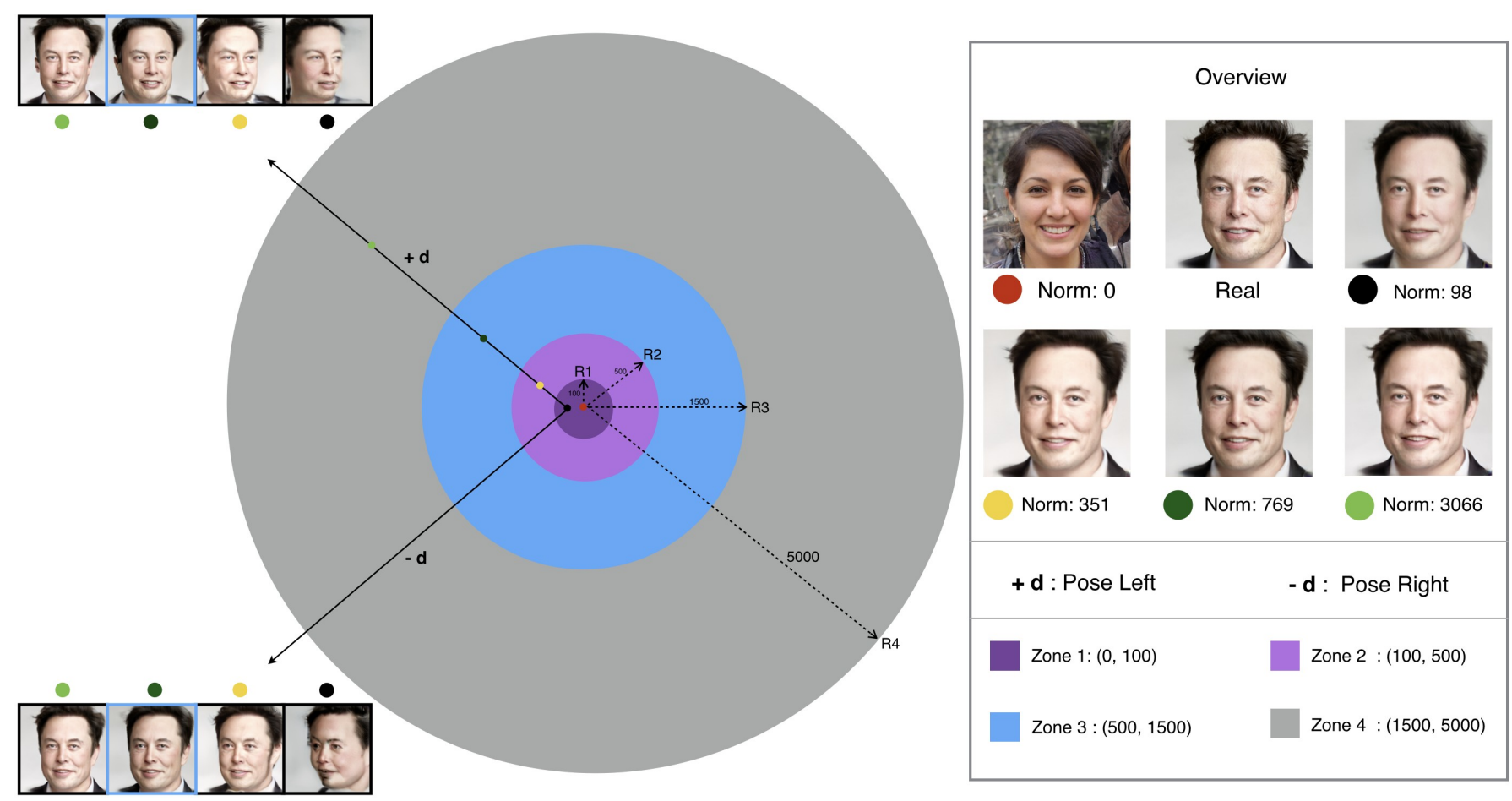

Figure 3: The above images are the morphing of real human faces. Based on StyleGAN [3], we utilise [11] that can embed real face images into style vectors $\left(w_{y}\right)$. Meanwhile, we use limited labels to classify face attributes, then we get the attribute vectors for real image morphing. Specifically, where image 'Red' (1st row) is from the attribute 'Red Hair', and image 'Hairs' (3rd row) is from the attribute 'Hairline'. Image 'Over Exposure' (2nd row) and Image 'Dark' (3rd row) are from the same attribute 'Exposure' (Opposite directions). Similarly, image 'Comedy' (2nd row) and image 'Arrogant' (2nd row) are also from the same attribute 'happy'.

\subsection{The Second Regularization for Attribute Vector}

The linear classification model needs to optimize $w_{d}$ and a bias $b$. In InterfaceGAN [14], the SVM is used to classify the $w_{d}$ with $d$ as Eq. 5

$$
\mathcal{L}_{w_{d}}=\arg \min _{w_{d}, b} \sum_{i=1}^{n} \max \left(0,1-d_{i}\left(w_{x_{i}}{ }^{T} w_{d}+b\right)\right)+\gamma\left\|w_{d}\right\|_{\rho=2}
$$

According to the implement of SVM [22], the default setting is $\gamma=1$ and $\rho=2\left(\ell_{2}-\right.$ norm $)$ in the regularization term. However, this method costs huge labels and time, but we are aiming to reduce these. Therefore, we consider a logistics regression (LR) classification as follows:

$$
\mathcal{L}_{w_{d}}=\arg \min _{w_{d}, b} \sum_{i=1}^{n} \log \left(\exp \left(-d_{i}\left(w_{x_{i}}{ }^{T} w_{d}+b\right)\right)+1\right)+\gamma\left\|w_{d}\right\|_{\rho=(1,2)}
$$

We found that the higher the classification accuracy of the above classifier, the higher performance of real image morphing. In addition, when classifying with LR, the performance of $\rho=1\left(\ell_{1}-\right.$ norm $)$ is slightly better than $\rho=2\left(\ell_{2}-\right.$ norm $)$, due to $\ell_{2}-$ norm produces more sparse $w_{d}$ that is more suitable for $w_{d}$ representation.

Based on these considerations, we added a trim operation. Let $w_{d}$ keep the features of $y$, while highlight the representation of $d$ as much as possible. The specific method is to set $\delta=[0.01,0.05]$ and $w_{d}=0$ when $w_{d}<\delta$, so that the each dimension of $w_{d}$ is larger than $\delta$. We can describe the whole method as the following algorithm 1 .

\section{Experiments}

Based on Python (3.7.3), the core implemented frameworks are PyTorch (1.5.1, with CUDA 10.2) and SciKit Learning (SKLearn, 0.24.2). The pre-trained models are StyleGAN $G$ (FFHQ-F) [3] and corresponding $E$ [11]. For style optimization on linear classifiers, we use LibSVM [22] to implement SVM $\left(\ell_{2}-\right.$ norm $)$ and Sega to implement LR $\left(\ell_{1}-\right.$ norm and $\ell_{2}-$ norm) [15, 16]. All linear classifiers are implemented by SKLearn 1 . We use a GPU Card (GeForce RTX 3090) to perform image embedding ( $y$ to $w_{y}$ ), and use a CPU card (Inter Core I5 10600KF) to perform attribute optimizing ( $d$ to $w_{d}$ ).

\subsection{Attributes and Classifiers}

We re-arranged two real face classifiers for collecting latent attribute 12,000 pairs $\left(w_{x}, d\right)$. One comes from Microsoft Cognitive Services API (StyleGAN-Encoder) ${ }^{2}$, and the other one comes from StyleGAN Binary classifier which be trained to detect attributes of CelebA-HQ 3 There are about 40 independent attributes in each of the two classifiers, but some attributes have

\footnotetext{
${ }^{1}$ SKLearn: https://scikit-learn.org/stable/modules/classes ${ }^{2}$ MS-Classifiers: https://azure.microsoft.com/en-us/services/ cognitive-services/face

${ }^{3}$ NV-Classifiers: https://github.com/NVlabs/stylegan
} 


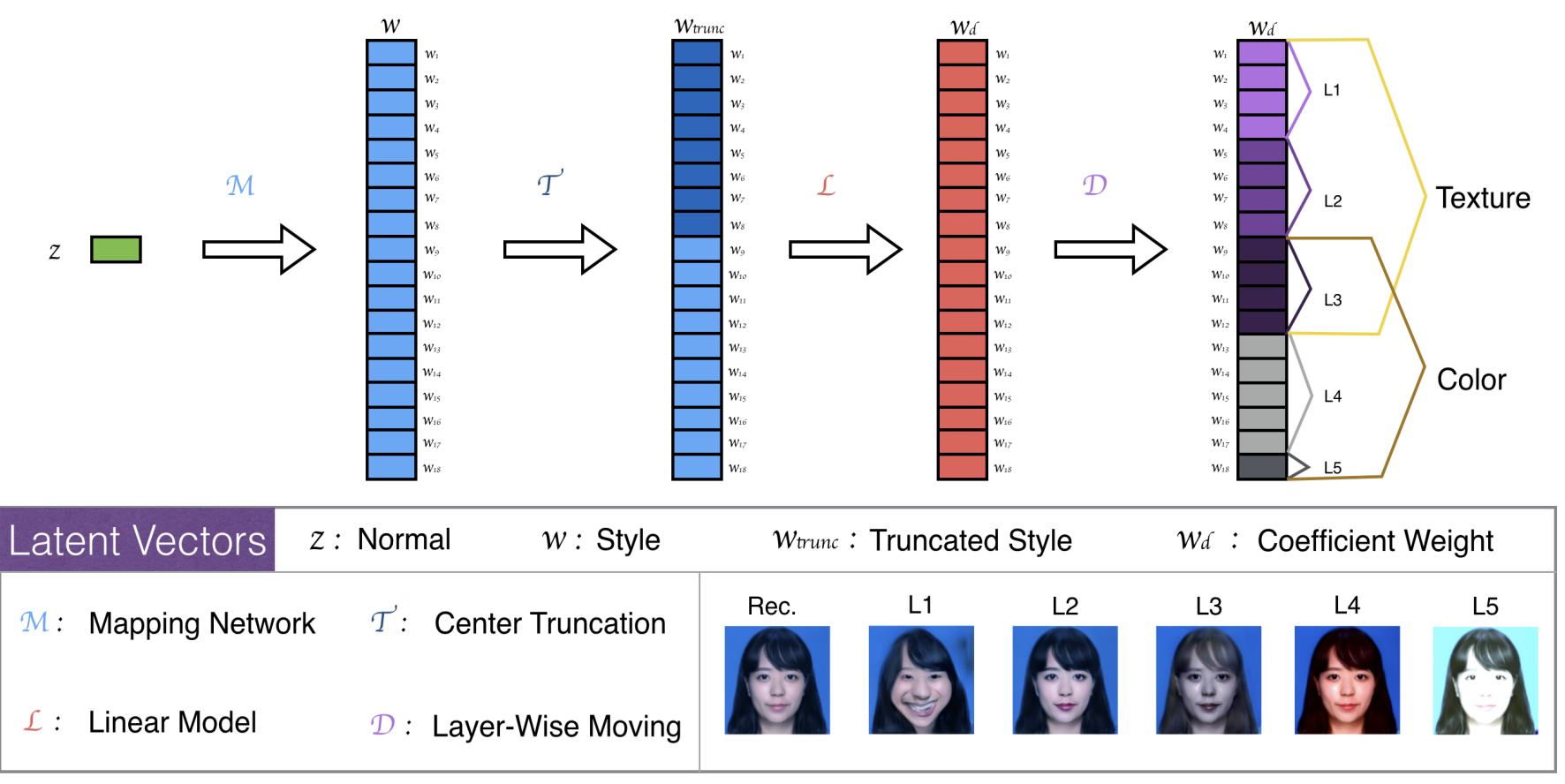

Figure 4: The pipeline of our method for optimizing $w_{d}, M$ (Mapping network) is a pre-trained mapping network from StyleGANs (contained 8 fully connected layers). $T$ (Center truncation) and $D$ (Layer-wise Moving) are vector operations. $L$ (Linear Model) is the method of logistic regression classification on $w_{d}$ with $d$.

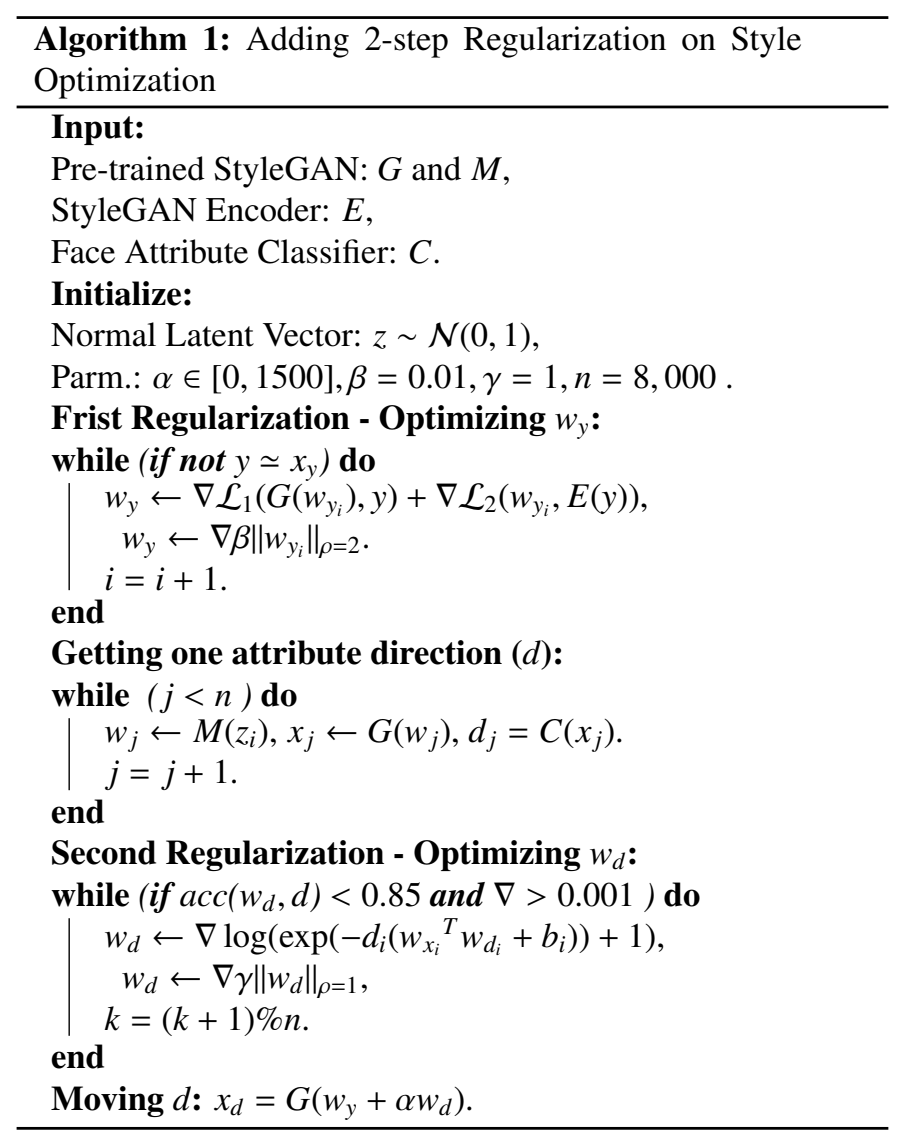

no samples or the attributes are not valid for image morphing, we did the integration and sorted out 32 attributes.

As shown in Tab. 1, these attributes are divided into different layer levels according to the different semantic levels. Each level contains 1-5 layers. From L1 to L5, the facial features represent texture to colour. Some of these attributes control the same semantic (Sim.), only the details are different in the same, such as Smile and Happiness, Age and Young, etc. In addition, there is a certain correlation between adjacent levels, and it can be necessary to increase or decrease 1-2 layers appropriately to achieve the better performance of fine-tuning the image morphing.

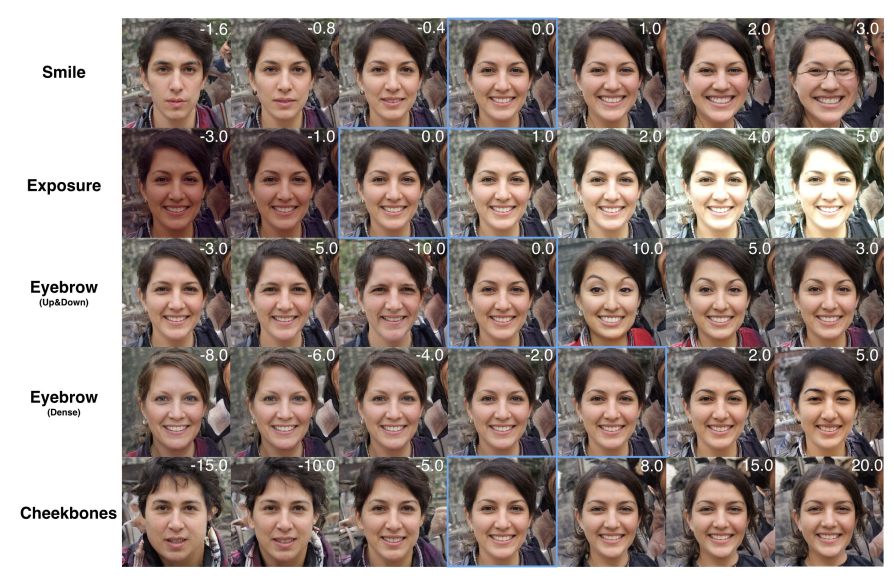

Figure 5: Moving $w_{d}$ from the original point $w_{y}($ Norm $=0)$. The distance parameter $\alpha$ (right top) is within $[0,20]$. 
Table 1:

Valid Attributes with Corresponding Layer Levels ( $\bullet$ denotes that the attribute is similar to the above one, and $\checkmark$ denotes the valid layer-levels).

\begin{tabular}{|c|l|c|c|c|c|c|c|}
\hline \multirow{2}{*}{ ID } & \multirow{2}{*}{ Name } & \multirow{2}{*}{ Sim. } & \multicolumn{5}{|c|}{ Semantic Level } \\
\hline & & & L1 & L2 & L3 & L4 & L5 \\
\hline 1 & Smile & & $\checkmark$ & & & & \\
\hline 2 & Happiness & $\bullet$ & $\checkmark$ & & & & \\
\hline 3 & Pose & & $\checkmark$ & & & & \\
\hline 4 & Gender & & $\checkmark$ & & & & \\
\hline 5 & Age & & $\checkmark$ & & & & \\
\hline 6 & Young & $\bullet$ & $\checkmark$ & & & & \\
\hline 7 & FaceShape (Oval) & & $\checkmark$ & & & & \\
\hline 8 & DoubleChin & $\bullet$ & $\checkmark$ & & & & \\
\hline 9 & Cheekbones & $\bullet$ & $\checkmark$ & & & & \\
\hline 10 & Glasses & & $\checkmark$ & $\checkmark$ & & & \\
\hline 11 & SunGlasses & $\bullet$ & $\checkmark$ & $\checkmark$ & & & \\
\hline 12 & ReadingGlasses & $\bullet$ & $\checkmark$ & $\checkmark$ & & & \\
\hline 13 & HairShape (Wavy) & & $\checkmark$ & $\checkmark$ & $\checkmark$ & & \\
\hline 14 & Bald & & & $\checkmark$ & & & \\
\hline 15 & Hairline & $\bullet$ & & $\checkmark$ & & & \\
\hline 16 & Bangs & $\bullet$ & & $\checkmark$ & & & \\
\hline 17 & LipsSize & & & $\checkmark$ & & & \\
\hline 18 & NoseSize & & & $\checkmark$ & & & \\
\hline 19 & Makeup (Face) & & & $\checkmark$ & $\checkmark$ & & \\
\hline 20 & Makeup (Eye) & $\bullet$ & & $\checkmark$ & $\checkmark$ & & \\
\hline 21 & Makeup (Lip) & $\bullet$ & & $\checkmark$ & $\checkmark$ & & \\
\hline 22 & Sadness & & & $\checkmark$ & $\checkmark$ & & \\
\hline 23 & Eyebrow (Loc.) & & & $\checkmark$ & $\checkmark$ & & \\
\hline 24 & Eyebrow (Dense) & & & $\checkmark$ & $\checkmark$ & & \\
\hline 25 & Moustache & & & $\checkmark$ & $\checkmark$ & & \\
\hline 26 & Beard & $\bullet$ & & $\checkmark$ & $\checkmark$ & & \\
\hline 27 & Sideburns & $\bullet$ & & $\checkmark$ & $\checkmark$ & & \\
\hline 28 & Goatee & $\bullet$ & & $\checkmark$ & $\checkmark$ & & \\
\hline 29 & BlackHair & & & & $\checkmark$ & $\checkmark$ & \\
\hline 30 & BlondHair & & & & $\checkmark$ & $\checkmark$ & \\
\hline 31 & RedHair & & & $\checkmark$ & $\checkmark$ & \\
\hline 32 & Exposure & & & & & $\checkmark$ \\
\hline
\end{tabular}

\subsection{Ablation Study on Regularization}

$w_{y}$ changes near the origin point are most sensitive (see Fig. 60 to attribute changes. That means when $w_{y}$ located in Norm $\in$ (10), Zone ${ }_{1}$, the attribute changes are more coupled than $w_{y}$ in the other zones (large Norm). As shown in Fig. 5, by moving the same $w_{d}$ (pose) with different $w_{y}$, where the black point located in Zone $_{1}$ is the most sensitive, and there is an easy coupling for image morphing, or even invalid. We test 32 real face images and observed that when $w_{y}$ falls into Zone 2 and $Z_{o n e_{3}}$, the attribute changes tend to be smooth, and we find that the better performance is $\mathrm{Zone}_{3}$ that can make face change more controllable. If $w_{y}$ falls into Zone $e_{4}$, moving $w_{d}$ will make little or even no change to face morphing, which is similar to GAN model collapse. It needs to add $\ell_{2}-$ norm in the $w_{y}$ optimization to make the $w_{y}$ falls into $Z_{o n e_{3}}$, i.e, the first step regularization, as shown in Eq. 3 . Next, we optimize $w_{d}$. We use $\ell_{1}-$ norm to

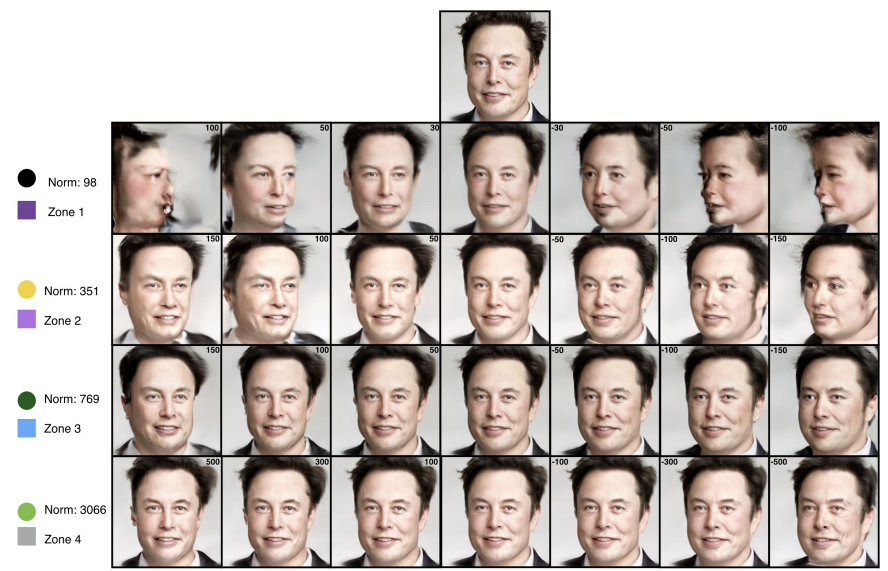

Figure 6: Moving the pose vector $\left(w_{d}\right)$ from different $w_{y}$ for the same person. $w_{y}$ filled into different Norm Zone. The image right top value is the $\alpha$ that is the distance parameter of $w_{d}$.

regularization (Eq. 6 with $\rho=1$ ), then layer-wise moving $w_{d}$ from the optimized $w_{y}$.

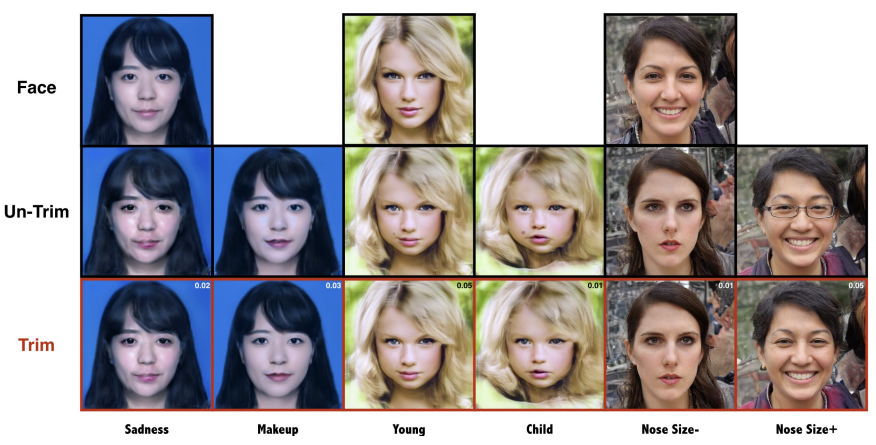

Figure 7: Trim Trick for $\ell_{1}-$ norm. The hair colour is coupled with sadness. The exposure is coupled with makeup (columns 1). The exposure is coupled with makeup (columns 2). Nevus is coupled with age (columns 3-4). Nose size (zoom out) is coupled with lips (columns 5). Nose size (zoom in) is coupled with eyeglasses (columns 6).

\subsection{Norm with Trim}

Our method could be enhanced by trimming $w_{d}$, due to both $w_{y}$ and $w_{d}$ are following the normal distribution. We attempt to optimize $w_{y}$ and $w_{d}$ to a similar distribution shape. Fig. 8 shows different Norm distributions of Musk's $w_{y}$. We found that the $w_{d}$ which optimized from LR $\left(\ell_{1}-\right.$ norm $)$ is same shape with $w_{y}$. As shown in Fig. 9, the first row shows there are different $w_{d}$ (age) distributions which optimized from 3 classifiers. The second row shows the trimmed $w_{d}$ distributions which optimized from $\operatorname{LR}\left(\ell_{1}-\right.$ norm $)$.

We report the trim performance on Fig. 7. We find that when trimmed and untrimmed $w_{d}$ move the same distance $\alpha$, the trimmed $w_{d}$ is more decoupled than untrimmed $w_{d}$.

\subsection{Compare with baseline}

We compared three different linear classifiers via the supervised manner, i.e, SVM Classifier (SVC) [14] $]^{4} \operatorname{LR}\left(\ell_{2}-\right.$ norm $)$

$\sqrt[4]{\text { https://github.com/genforce/interfacegan.git }}$ 

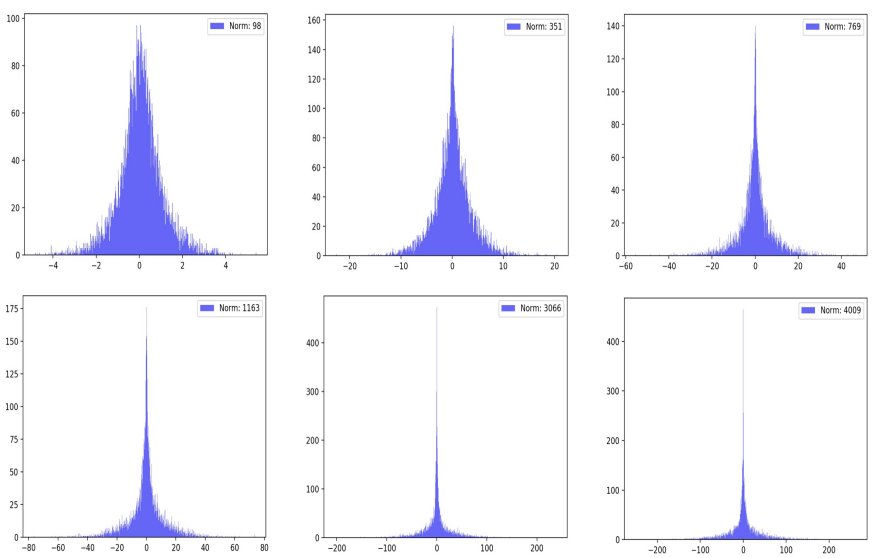

Figure 8: Norm distributions on different $w_{y}$ for the same person, $y$ (Musk)
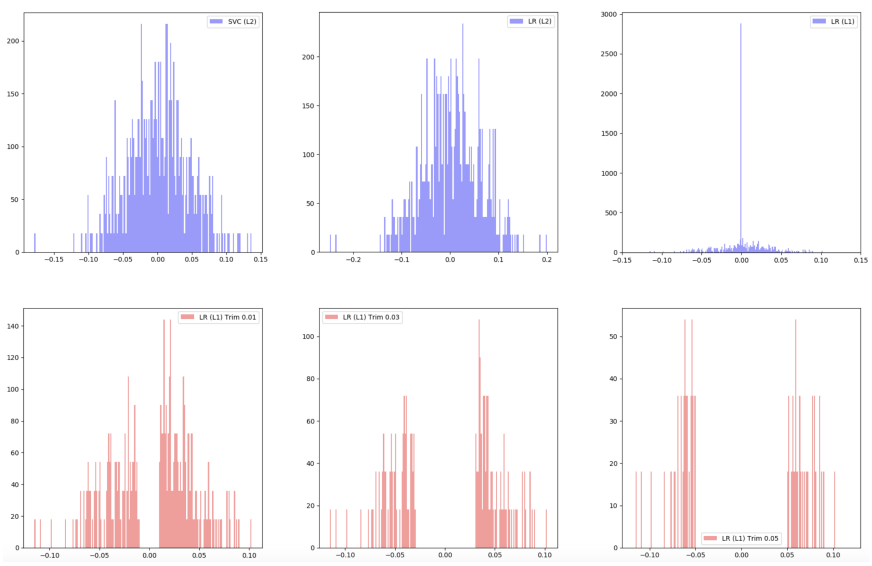

Figure 9: Different distribution of $w_{d}$ and trim on $\operatorname{LR}\left(\ell_{1}-\right.$ norm $)$.

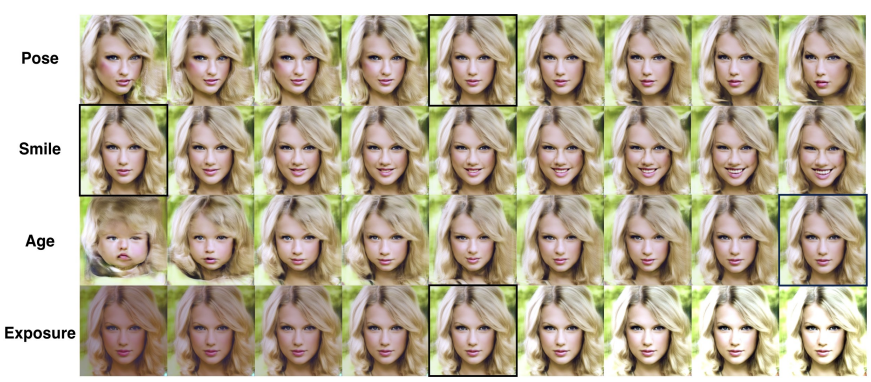

Figure 10: Real Face Morphing based on 2-Step Regularization. That perform as animations for frame by frame.

Table 2:

Comparing Unsupervised with Supervised Methods.

\begin{tabular}{|c|c|c|c|c|c|c|}
\hline & \multirow{2}{*}{ Method } & \multicolumn{3}{|c|}{ Training Cost } & \multicolumn{2}{c|}{ Attributes } \\
\cline { 3 - 7 } & & Samples & Labels & Time(min) & Class. & Ind. \\
\hline GANSpace [12] & PCA & $30 \mathrm{~K}$ & None & $30-45$ & 100 & 17 \\
\hline EigenGAN [13] & PCA & $203 \mathrm{~K}$ & None & - & 36 & 16 \\
\hline InterfaceGAN [14] & SVM & $500 \mathrm{~K}$ & $20 \mathrm{~K}$ & $80-175$ & 40 & - \\
\hline Ours & LR & $20 \mathrm{~K}$ & $12 \mathrm{~K}$ & $3-10$ & 80 & $\mathbf{1 9}$ \\
\hline
\end{tabular}

[23] 5 with our method (LR, $\ell_{1}-$ norm). Depending on the increasing number of training pairs $\left(w_{d}\right.$ and $d$ ), we measure 33 attributes upon the time cost (Sec.) and classification accuracy (Acc.). We report 3 typical results in Fig. 11 and more details on Table. 3. We observed that the accuracy turns stable after the training samples exceed $8 \mathrm{~K}$. However, the time cost has increased significantly with samples increasing, especially on SVC. For trading off the cost and performance, we chose $8 \mathrm{k}$ samples for optimizing $w_{d}$. Compared with other methods, our method has slightly improved accuracy and smaller operation time costs.

We also compared the unsupervised methods (GANSpace [12], EigenGAN [13]). As shown in Table 3, the unsupervised methods are based on principal component analysis PCA and require many training samples and time cost. GANSpace [12] gets $w_{d}$ through the $w$ optimizing and find the main components. EigenGAN needs to train GAN models with orthogonal $w_{d}$ to represent semantic $d$. Compared to our method in a supervised manner, InterfaceGAN [14] needs huge training costs (time and samples). Indeed, GANSpace and EigenGAN also require labour costs to explore the semantics of $w_{d}$. Many principal components are not suitable for face morphing. Using a few training tags to find effective independent attributes, our method has a few advantages. Differently from previous works of finding semantic attributes, we also pay more attention to the smoothness of attribute changes, so that we can make the frame morphing as frame-by-frame animations [24] (see Fig. 10].

\section{Conclusion}

We propose a novel method for real face morphing based on the distribution pattern of latent space. We use a two-step regularization for real face embedding and attribute searching. First, we find the face latent vector $w_{y}$ by adding $\ell_{2}-$ norm regularization. Then, we find the attribute latent vector $w_{d}$ by adding $\ell_{1}-$ norm regularization. Finally, we move layer-wise $w_{d}$ from $w_{y}$ for image morphing. In addition, we improve the face morphing effect by $w_{d}$ trim. Compared to previous works, we also integrate two attribute classifiers to find more semantic attributes. Our performance is smoother in controlling face changes, even up to the standard of animation. Our approach offers a lower-cost alternative that is also faster and easier to deploy.

\section{Limitations and Further Works}

Compared to other supervised methods, our method utilises fewer training samples with labels, but it is still completed under the manner of supervised learning. Although there are methodologies that find the semantic attributes through an unsupervised approach, many shortcomings remain. For example, attributes need to be found in the main independent components, and many attributes are repetitive or even invalid. The number of detected attributes is very small (fewer than

$\sqrt[5]{\text { https://github.com/Puzer/stylegan-encoder }}$ 

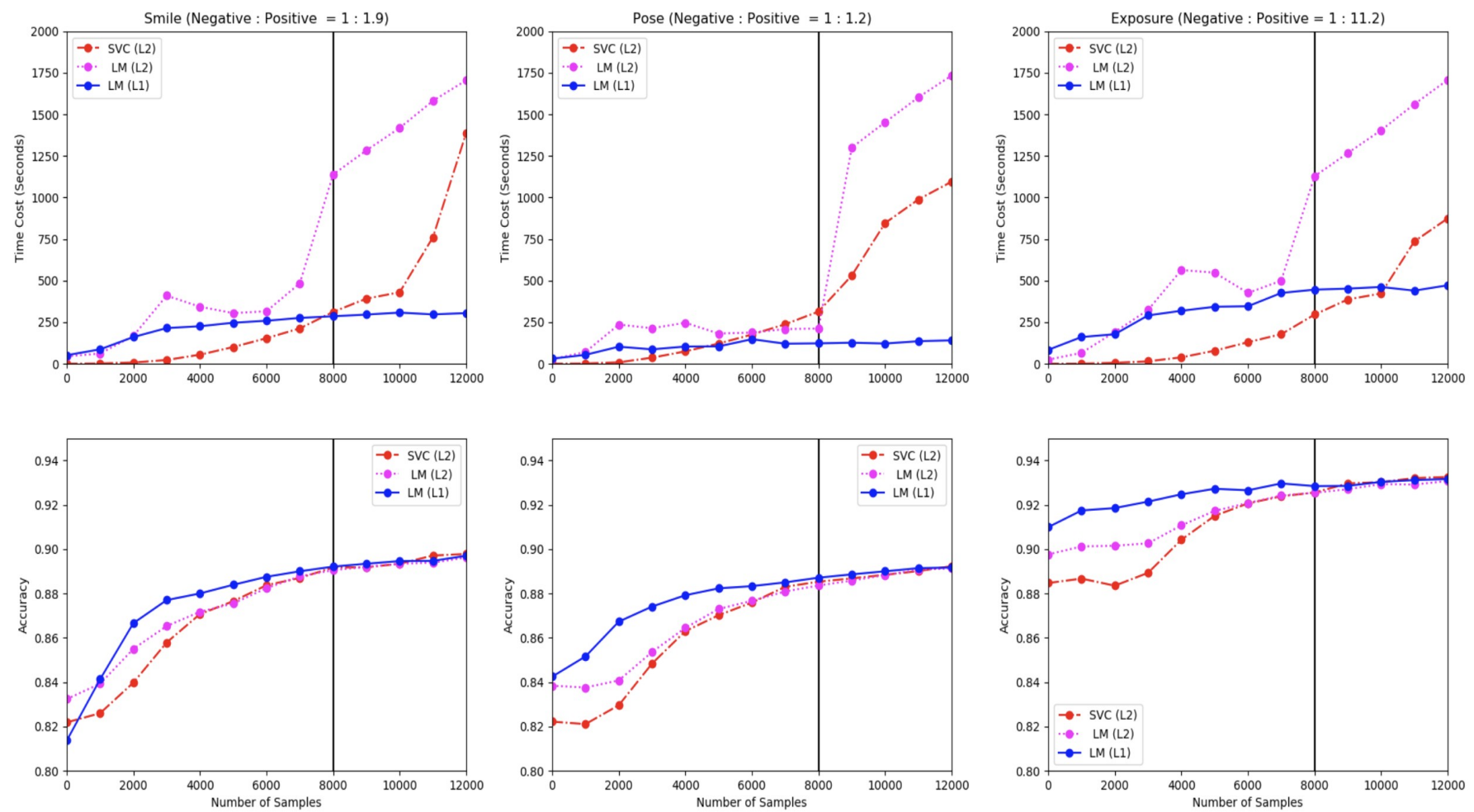

Figure 11: Comparison of supervision methods, the first row shows the cost times. The second row shows the accuracies.

20). Next, we intend to explore semantic attributes in a selfsupervised manner under the interpretability and representation of the neural networks. Beyond the existing methods, we aim to develop new methods for controlling visual generation.

\section{CRediT authorship contribution statement}

Cheng Yu: Conceptualization, Methodology, Software, Writing - Original Draft, Resource, Data curation. Wenmin Wang: Formal analysis, Conceptualization, Project administration, Funding acquisition, Supervision, Writing - Review \& Editing. Honglei LI: Validation, Investigation, Writing - Review \& Editing. Roberto Bugiolacchi: Writing - Review \& Editing, Investigation, Visualization.

\section{Declaration of Competing Interest}

We confirm that the current version of the manuscript has been read and approved by all named authors and that there are no other persons who satisfied the criteria for authorship but are not listed. We confirm that the order of authors listed in the manuscript has been approved by all of us. There are no known conflicts of interest associated with this publication and there has been no significant financial support for this work that could have influenced its outcome.

\section{Acknowledgements}

This work was supported by the Science and Technology Development Fund (FDCT) of Macau (0016/2019/A1). We will arrange to release the code on GitHub.

\section{References}

[1] I. Goodfellow, J. Pouget-Abadie, M. Mirza, B. Xu, D. Warde-Farley, S. Ozair, A. Courville, Y. Bengio, Generative adversarial nets, in: Proc. Int. Conf. Neural Inf. Process. Syst. (NIPS), Vol. 27, 2014, pp. 2672-2680.

[2] Z. Liu, P. Luo, X. Wang, X. Tang, Deep learning face attributes in the wild, in: Proc. IEEE Int. Conf. Comput. Vis. (ICCV), 2015, pp. 37303738 .

[3] T. Karras, S. Laine, T. Aila, A style-based generator architecture for generative adversarial networks, in: Proc. IEEE Conf. Comput. Vis. Pattern Recognit. (CVPR), 2019.

[4] A. Radford, L. Metz, S. Chintala, Unsupervised representation learning with deep convolutional generative adversarial networks, in: Int. Conf. Learn. Represent. (ICLR), 2016.

[5] T. Karras, T. Aila, S. Laine, J. Lehtinen, Progressive growing of gans for improved quality, stability, and variation, in: Int. Conf. Learn. Represent. (ICLR), 2018.

[6] A. Brock, J. Donahue, K. Simonyan, Large scale GAN training for high fidelity natural image synthesis, in: Int. Conf. Learn. Represent. (ICLR), 2019.

[7] T. Karras, S. Laine, M. Aittala, J. Hellsten, J. Lehtinen, T. Aila, Analyzing and improving the image quality of stylegan, in: Proc. IEEE Conf. Comput. Vis. Pattern Recognit. (CVPR), 2020, pp. 8107-8116.

[8] T. Karras, M. Aittala, S. Laine, E. Härkönen, J. Hellsten, J. Lehtinen, T. Aila, Alias-free generative adversarial networks, arXiv preprint abs/2106.12423.

[9] R. Abdal, Y. Qin, P. Wonka, Image2stylegan: How to embed images into the stylegan latent space?, in: Proc. IEEE Int. Conf. Comput. Vis. (ICCV), 2019, pp. 4431-4440.

[10] C. Yu, W. Wang, Fast transformation of discriminators into encoders using pre-trained gans, Pattern Recognition Letters 153 (2022) 92-99.

[11] C. Yu, W. Wang, Adaptable gan encoders for image reconstruction via multi-type latent vectors with two-scale attentions (2021). $\operatorname{arXiv:2108.}$ 10201 
[12] E. Härkönen, A. Hertzmann, J. Lehtinen, S. Paris, Ganspace: Discovering interpretable GAN controls, in: Proc. Int. Conf. Neural Inf. Process. Syst. (NIPS), 2020

[13] Z. He, M. Kan, S. Shan, Eigengan: Layer-wise eigen-learning for gans, in: Proc. IEEE Int. Conf. Comput. Vis. (ICCV), 2021.

[14] Y. Shen, C. Yang, X. Tang, B. Zhou, Interfacegan: Interpreting the disentangled face representation learned by gans, IEEE Trans. on Pattern Analysis and Machine Intelligence (TPAMI)

[15] Jerome, Friedman, Trevor, Hastie, Rob, Tibshirani, Regularization paths for generalized linear models via coordinate descent., Journal of statistical software 33 (1) (2010) 1-22.

[16] S. Kim, K. Koh, M. Lustig, S. P. Boyd, D. Gorinevsky, An interior-point method for large-scale \$ $\backslash$ ell_1\$-regularized least squares, J. Sel. Topics Signal Processing 1 (4) (2007) 606-617.

[17] C. Yang, Y. Shen, B. Zhou, Semantic hierarchy emerges in deep generative representations for scene synthesis, Int. J. Comput. Vis. 129 (5) (2021) 1451-1466.

[18] D. Bau, J. Zhu, H. Strobelt, B. Zhou, J. B. Tenenbaum, W. T. Freeman, A. Torralba, GAN dissection: Visualizing and understanding generative adversarial networks, in: Int. Conf. Learn. Represent. (ICLR), 2019.

[19] F. Yu, Y. Zhang, S. Song, A. Seff, J. Xiao, LSUN: construction of a largescale image dataset using deep learning with humans in the loop, arXiv preprint abs/1506.03365.

[20] J. Zhu, Y. Shen, D. li Zhao, B. Zhou, In-domain gan inversion for real image editing, in: Europ. Conf. Comput. Vis. (ECCV), 2020.

[21] S. Pidhorskyi, D. A. Adjeroh, G. Doretto, Adversarial latent autoencoders, in: Proc. IEEE Conf. Comput. Vis. Pattern Recognit. (CVPR), 2020.

[22] C. Chang, C. Lin, LIBSVM: A library for support vector machines, ACM Trans. Intell. Syst. Technol. 2 (3) (2011) 27:1-27:27.

[23] D. Nikitko, Stylegan encoder for official tensorflow implementation, https://github.com/Puzer/stylegan-encoder (2019).

[24] C. Yu, W. Wang, J. Yan, Self-supervised animation synthesis through adversarial training, IEEE Access 8 (2020) 128140-128151. 
Table 3:

Comparing Supervised Methods.

\begin{tabular}{|c|c|c|c|c|c|c|c|c|c|c|c|c|c|c|c|}
\hline Attri. & Model & & $0.5 \mathrm{~K}$ & $1 \mathrm{~K}$ & $2 \mathrm{~K}$ & $3 \mathrm{~K}$ & $4 \mathrm{~K}$ & $5 \mathrm{~K}$ & $6 \mathrm{~K}$ & $7 \mathrm{~K}$ & $8 \mathrm{~K}$ & $9 \mathrm{~K}$ & $10 \mathrm{~K}$ & $11 \mathrm{~K}$ & $12 \mathrm{~K}$ \\
\hline \multirow{8}{*}{ Exposure } & \multirow{2}{*}{ Total } & Neg. & 40 & 79 & 155 & 237 & 322 & 413 & 501 & 574 & 655 & 738 & 819 & 906 & 983 \\
\hline & & Pos. & 460 & 921 & 1845 & 2763 & 3768 & 4587 & 5499 & 6426 & 7345 & 8262 & 9181 & 10094 & 11017 \\
\hline & \multirow{2}{*}{ SVC [14] } & Sec. & 1 & 1 & 6 & 15 & 39 & 79 & 130 & 178 & 296 & 387 & 423 & 736 & 874 \\
\hline & & Acc. & 0.885 & 0.887 & 0.884 & 0.889 & 0.904 & 0.915 & 0.921 & 0.924 & 0.926 & 0.930 & 0.930 & 0.932 & 0.933 \\
\hline & \multirow{2}{*}{ LR (L2) [23] } & Sec. & 25 & 66 & 191 & 324 & 564 & 548 & 426 & 500 & 1127 & 1267 & 1404 & 1561 & 1707 \\
\hline & & Acc. & 0.898 & 0.901 & 0.902 & 0.903 & 0.911 & 0.917 & 0.921 & 0.924 & 0.925 & 0.927 & 0.929 & 0.929 & 0.931 \\
\hline & \multirow{2}{*}{ LR (L1) [Ours] } & Sec. & 84 & 161 & 178 & 291 & 319 & 343 & 346 & 426 & 446 & 452 & 462 & 440 & 472 \\
\hline & & Acc. & 0.910 & 0.917 & 0.919 & 0.921 & 0.925 & 0.927 & 0.926 & 0.930 & 0.928 & 0.929 & 0.930 & 0.931 & 0.932 \\
\hline \multirow{8}{*}{ Smile } & \multirow{2}{*}{ Total } & Neg. & 170 & 332 & 677 & 1011 & 1328 & 1659 & 1986 & 2327 & 2674 & 3024 & 3370 & 3709 & 4069 \\
\hline & & Pos. & 330 & 668 & 1323 & 1989 & 2672 & 3341 & 4014 & 4673 & 5326 & 5976 & 6630 & 7291 & 7931 \\
\hline & \multirow{2}{*}{ SVC } & Sec. & 1 & 2 & 8 & 23 & 55 & 101 & 154 & 213 & 312 & 392 & 430 & 761 & 1389 \\
\hline & & Acc. & 0.822 & 0.826 & 0.840 & 0.858 & 0.871 & 0.877 & 0.884 & 0.887 & 0.892 & 0.892 & 0.893 & 0.897 & 0.898 \\
\hline & \multirow{2}{*}{ LR (L2) } & Sec. & 45 & 62 & 168 & 410 & 343 & 305 & 316 & 484 & 1138 & 1283 & 1418 & 1581 & 1705 \\
\hline & & Acc. & 0.832 & 0.839 & 0.855 & 0.865 & 0.872 & 0.876 & 0.882 & 0.888 & 0.891 & 0.892 & 0.893 & 0.894 & 0.896 \\
\hline & \multirow{2}{*}{ LR (L1) } & Sec. & 52 & 87 & 161 & 215 & 226 & 247 & 259 & 276 & 286 & 296 & 308 & 297 & 305 \\
\hline & & Acc. & 0.814 & 0.841 & 0.867 & 0.877 & 0.880 & 0.884 & 0.888 & 0.890 & 0.892 & 0.893 & 0.895 & 0.895 & 0.897 \\
\hline \multirow{8}{*}{ Pose } & \multirow{2}{*}{ Total } & Neg. & 223 & 450 & 883 & 1352 & 1818 & 2277 & 2746 & 3174 & 3646 & 4093 & 4545 & 4963 & 5421 \\
\hline & & Pos. & 277 & 550 & 1117 & 1648 & 2182 & 2723 & 3254 & 3826 & 4354 & 4907 & 5455 & 6037 & 6579 \\
\hline & & Sec. & 1 & 2 & 9 & 37 & 75 & 122 & 176 & 238 & 314 & 531 & 846 & 988 & 1095 \\
\hline & & Acc. & 0.822 & 0.821 & 0.830 & 0.848 & 0.863 & 0.870 & 0.876 & 0.883 & 0.885 & 0.887 & 0.888 & 0.890 & 0.892 \\
\hline & & Sec. & 28 & 71 & 236 & 214 & 247 & 182 & 188 & 209 & 212 & 1300 & 1452 & 1602 & 1733 \\
\hline & & Acc. & 0.838 & 0.837 & 0.841 & 0.854 & 0.865 & 0.873 & 0.877 & 0.881 & 0.884 & 0.886 & 0.888 & 0.890 & 0.891 \\
\hline & & Sec. & 31 & 54 & 103 & 87 & 104 & 105 & 148 & 121 & 123 & 127 & 122 & 136 & 141 \\
\hline & & Acc. & 0.843 & 0.852 & 0.867 & 0.874 & 0.879 & 0.882 & 0.883 & 0.885 & 0.887 & 0.889 & 0.890 & 0.891 & 0.892 \\
\hline
\end{tabular}

